\title{
Polynomial invariants for discrimination and classification of four-qubit entanglement
}

\author{
Oliver Viehmann \\ Physics Department, Arnold Sommerfeld Center for Theoretical Physics, and Center for NanoScience, Ludwig-Maximilians-Universität, \\ Theresienstraße 37, D-80333 München, Germany \\ Christopher Eltschka \\ Institut für Theoretische Physik, Universität Regensburg, D-93040 Regensburg, Germany \\ Jens Siewert \\ Departamento de Química Física, Universidad del País Vasco-Euskal Herriko Unibertsitatea, Apdo. 644, ES-48080 Bilbao, Spain, and \\ Ikerbasque, Basque Foundation for Science, Alameda Urquijo 36, ES-48011 Bilbao, Spain
}

(Received 21 November 2010; published 31 May 2011)

\begin{abstract}
The number of entanglement classes in stochastic local operations and classical communication (SLOCC) classifications increases with the number of qubits and is already infinite for four qubits. Criteria for explicitly discriminating and classifying pure states of four and more qubits are highly desirable and therefore at the focus of intense theoretical research. We develop a general criterion for the discrimination of pure $N$-partite entangled states in terms of polynomial SL $(d, \mathbb{C})^{\otimes N}$ invariants. By means of this criterion, existing SLOCC classifications of four-qubit entanglement are reproduced. Based on this we propose a polynomial classification scheme in which entanglement types are identified through "tangle patterns." This scheme provides a practicable way to classify states of arbitrary multipartite systems. Moreover, the use of polynomials induces a corresponding quantification of the different types of entanglement.
\end{abstract}

DOI: 10.1103/PhysRevA.83.052330

PACS number(s): 03.67.Mn

\section{INTRODUCTION}

In essence, entanglement comprises the nonlocal correlations in a multiparty system, i.e., the ones which cannot be enhanced on average by applying stochastic local operations and classical communication (SLOCC) to the quantum state. Properties of the state that remain unchanged under SLOCC may serve to characterize the entanglement type and, thus, to build an SLOCC classification for the states of a multipartite quantum system, e.g., Refs. [1-14].

On the other hand, there are well-known polynomial functions of the coefficients of pure quantum states which play an important role as entanglement measures [1,5,15-21]. Such measures have to be homogeneous and invariant under local special linear transformations $[1,16]$. For pure two-qubit and three-qubit states, concurrence [17] and three-tangle [18] are the unique polynomials of this kind [22].

Presumably the most interesting consequence deriving from the properties of invariant polynomials in the cases of two and three qubits is that they impose an SLOCC classification of entangled states [1]. There is only one type of entanglement for two qubits and the concurrence is nonvanishing exactly for the entangled states. In multipartite system, different types of entanglement may occur. For example, pure three-qubit entangled states may belong to the class of GHZ-type states or to the class of $W$-type states, where the three-tangle is nonzero exactly for the Greenberger-Horne-Zeilinger (GHZ) class [1]. That is, concurrence and three-tangle quantify class-specific entanglement.

For four and more qubits the number of SLOCC classes is infinite [1]. Therefore, the general idea of any SLOCC classification is to arrange the representatives of the infinitely many classes into a finite number of sets according to some SLOCC-invariant criterion, such as the Schmidt measure $[1,23]$, the degeneracy configuration [11], or the structure of the right singular subspace of the state coefficient matrix [8]. Obviously, all classifications comprise exactly the same classes-merely the arrangement into sets (which, henceforth, we call families) is different. The families are defined by the representative states they contain, thus providing a coarse graining to the SLOCC classes. At least one family comprises an infinite number of them.

In the existing classifications of pure four-qubit states it is not easy to determine to which class or family a given arbitrary state belongs, nor are they readily generalized to more complicated Hilbert spaces. Whether there exists a general relation between polynomial invariants and SLOCC classification analogous to the cases of two and three qubits is also still unknown. The only efforts touching upon this question were made in Refs. [9,10,13], however without a compelling answer. The aim of this paper is to fill these gaps and to bring qualitative and quantitative aspects of entanglement theory in line. To begin, we introduce a strong sufficient criterion for distinguishing SLOCC classes of arbitrary multipartite states based on polynomial invariants. We illustrate its application by extending earlier findings by Li et al. [13]. Subsequently, we show that certain sets of four-qubit polynomial invariants generate families of states which match the classifications of symmetric and of general four-qubit states due to Bastin et al. [11] and Lamata et al. [8], respectively. As our main result, we then propose a polynomial-based SLOCC classification which we exemplify explicitly for four qubits, but whose generalization to other multipartite systems is straightforward.

\section{POLYNOMIAL DISCRIMINATION CRITERION FOR PURE MULTIPARTITE STATES}

Two pure quantum states $\psi^{(N)}$ and $\tilde{\psi}^{(N)}$ of an $N$-partite Hilbert space $\mathcal{H}^{(N)}=\mathcal{H}_{1} \otimes \cdots \otimes \mathcal{H}_{N}$ are interconvertible with a nonzero chance by means of SLOCC if and only if 
there exist invertible operators $J_{1}, \ldots, J_{N}$ with $J_{k} \in \operatorname{GL}\left(d_{k}, \mathbb{C}\right)$ acting on the local Hilbert spaces $\mathcal{H}_{k}$ (of dimension $d_{k}$ ) such that [1]

$$
\left|\tilde{\psi}^{(N)}\right\rangle=J_{1} \otimes \cdots \otimes J_{N}\left|\psi^{(N)}\right\rangle .
$$

Throughout this paper we consider unnormalized vectors $\psi^{(N)}$. By means of Eq. (1) SLOCC interconvertibility imposes an equivalence relation on the set of all vectors of $\mathcal{H}^{(N)}$. The SLOCC equivalence classes (also called orbits) are sets of (SLOCC-interconvertible, i.e., SLOCC-equivalent) states with equivalent multipartite entanglement in the sense that, under SLOCC, the same tasks can be performed with them.

Suppose $\mathcal{P}_{[i]}$ and $\mathcal{P}_{[j]}^{\prime}$ are homogeneous functions of degrees $i$ and $j$ of the states in a Hilbert space $\mathcal{H}^{(N)}$ that are invariant under $\operatorname{SL}\left(d_{k}, \mathbb{C}\right)^{\otimes N}$ transformations, where $d_{k}=$ $\operatorname{dim}\left\{\mathcal{H}_{k}\right\}$. Then, for integers $m, n$ with $i m=j n$, and a fixed state $\psi^{(N)}$, a complex number $\eta$ exists such that

$$
\left[\mathcal{P}_{[i]}\left(\psi^{(N)}\right)\right]^{m}=\eta\left[\mathcal{P}_{[j]}^{\prime}\left(\psi^{(N)}\right)\right]^{n} .
$$

Here $\eta$ is unique and invariant under SLOCC transformations on $\psi^{(N)}$ as long as $\mathcal{P}_{[j]}^{\prime}$ is different from zero. That is, for $\tilde{\psi}^{(N)}=J_{1} \otimes \cdots \otimes J_{N} \psi^{(N)}$ we have also $\left[\mathcal{P}_{[i]}\left(\tilde{\psi}^{(N)}\right)\right]^{m}=$ $\eta\left[\mathcal{P}_{[j]}^{\prime}\left(\tilde{\psi}^{(N)}\right)\right]^{n}$. The ratio of homogeneous $\operatorname{SL}\left(d_{k}, \mathbb{C}\right)^{\otimes N}$ invariants of the same degree is invariant under SLOCC. Therefore, for two SLOCC-equivalent states the ratio of two arbitrary invariants must be the same. The spirit of this criterion has been applied before $[13,15]$, however without emphasizing its generality.

An important consequence is that from two independent invariants (which for more than three qubits can always be found [5,24]) one can construct an invariant that vanishes for a given SLOCC class due to

$$
\mathcal{P}_{[i]}^{m}-\eta \mathcal{P}_{[j]}^{\prime n}=0
$$

In the following, we will focus exclusively on polynomial invariants and the four-qubit Hilbert space, since in that case all generators of the algebra of polynomial invariants [15,24] are known. Following the notation of Ref. [24] we define

$$
\begin{gathered}
\left(\left(A_{1} \bullet \cdots \bullet A_{N}\right)\right)=\left\langle\psi^{*} \mid A_{1} \psi\right\rangle \cdots\left\langle\psi^{*} \mid A_{N} \psi\right\rangle, \\
\sigma_{\mu} \bullet \sigma^{\mu}=\sum_{\mu=0}^{3} g_{\mu} \cdot \sigma_{\mu} \bullet \sigma_{\mu}
\end{gathered}
$$

for operators $A_{i}$ that act on the Hilbert space of $\psi$, the Pauli matrices $\left(\sigma_{0}, \sigma_{1}, \sigma_{2}, \sigma_{3}\right)=\left(\mathbb{1}_{2}, \sigma_{x}, \sigma_{y}, \sigma_{z}\right)$, and $\left(g_{0}, g_{1}, g_{2}, g_{3}\right):=$ $(-1,1,0,1)$. The $\bullet$ symbol denotes a tensor product that refers to copies of the same state whereas we do not specify tensor products between the parties: $\cdots \sigma_{\mu} \sigma_{v} \cdots \equiv \cdots \sigma_{\mu} \otimes \sigma_{v} \cdots$. As generators for the $\operatorname{SL}(2, \mathbb{C})^{\otimes 4}$-invariant polynomials we may choose, e.g.,

$$
\begin{gathered}
\mathcal{A}_{[2]}=\left(\left(\sigma_{2} \sigma_{2} \sigma_{2} \sigma_{2}\right)\right), \\
\mathcal{B}_{[4]}^{I}=\left(\left(\sigma_{\mu} \sigma_{\nu} \sigma_{2} \sigma_{2} \bullet \sigma^{\mu} \sigma^{\nu} \sigma_{2} \sigma_{2}\right)\right), \\
\mathcal{B}_{[4]}^{I I}=\left(\left(\sigma_{\mu} \sigma_{2} \sigma_{\nu} \sigma_{2} \bullet \sigma^{\mu} \sigma_{2} \sigma^{\nu} \sigma_{2}\right)\right), \\
\mathcal{C}_{[6]}=\left(\left(\sigma_{\mu} \sigma_{\nu} \sigma_{2} \sigma_{2} \bullet \sigma^{\mu} \sigma_{2} \sigma_{\lambda} \sigma_{2} \bullet \sigma_{2} \sigma^{\nu} \sigma^{\lambda} \sigma_{2}\right)\right) .
\end{gathered}
$$

This set is complete. The generator $\mathcal{A}_{[2]}$ is the well-known fourconcurrence [19] and $\mathcal{C}_{[6]}$ was introduced in Ref. [5]. We define
$\mathcal{B}_{[4]}^{I I I}$ via the sum rule [24] $\mathcal{B}^{I}+\mathcal{B}^{I I}+\mathcal{B}^{I I I}=3 \mathcal{A}^{2}$ (where we omit the subscript indicating the homogeneous degree). Note that the polynomials $\mathcal{B}^{j}$ are not invariant under qubit permutation.

The knowledge of all generators allows us to exhaustively exploit our criterion to distinguish the SLOCC classes of two four-qubit states $\psi^{(4)}$ and $\tilde{\psi}^{(4)}$. We introduce the abbreviations $\mathcal{A}\left(\psi^{(4)}\right)=\alpha, \mathcal{A}\left(\tilde{\psi}^{(4)}\right)=\tilde{\alpha}, \dot{\mathcal{B}}^{I}\left(\psi^{(4)}\right)=\beta_{1}, \ldots, \mathcal{C}\left(\tilde{\psi}^{(4)}\right)=\tilde{\gamma}$. Our criterion leads to the following equations that can be checked in order to decide whether the states $\psi^{(4)}$ and $\tilde{\psi}^{(4)}$ may belong to the same SLOCC class:

$$
\left(\frac{\alpha}{\tilde{\alpha}}\right)^{2}=\frac{\beta_{1}}{\tilde{\beta}_{1}}=\frac{\beta_{2}}{\tilde{\beta}_{2}}, \quad\left(\frac{\alpha}{\tilde{\alpha}}\right)^{3}=\frac{\gamma}{\tilde{\gamma}} .
$$

If, e.g., $\tilde{\alpha}$ is zero, its counterpart $\alpha$ must be zero as well; otherwise $\psi^{(4)}$ and $\tilde{\psi}^{(4)}$ cannot be SLOCC equivalent. In contrast, if all equations in (7) hold, the states are not necessarily SLOCC interconvertible.

For example, Li et al. [13] studied states of the $G_{a b c d}$ family from Ref. [3] with $b=c=0$ and $a, d \neq 0$. They concluded that this family can be split into three subfamilies, two of which contain only a single SLOCC class. Whether this is also the case for the third subfamily (A1.3) in Ref. [13] remained an open question. We can easily negate it by means of our criterion as formulated in equations (7). The representative of $G_{a b c d}$ for $b=c=0$ is

$$
\begin{aligned}
\left|\psi_{a b c d}\right\rangle= & \frac{(a+d)}{2}(|0000\rangle+|1111\rangle) \\
& +\frac{(a-d)}{2}(|0011\rangle+|1100\rangle)
\end{aligned}
$$

and the generators in Eq. (6) yield

$$
\begin{gathered}
\alpha=a^{2}+d^{2}, \quad \beta_{1}=3 a^{4}-2 a^{2} d^{2}+3 d^{4}, \\
\beta_{2}=4 a^{2} d^{2}, \quad \gamma=-4 a^{2} d^{2}\left(a^{2}+d^{2}\right) .
\end{gathered}
$$

One sees that Eqs. (7) can be violated (e.g., $a=d=\tilde{a}=$ $1, \tilde{d}=\sqrt{2}$ ). Consequently, subfamily A1.3 contains more than one SLOCC class.

\section{POLYNOMIAL CLASSIFICATION OF SYMMETRIC FOUR-QUBIT STATES}

Now we turn to a classification of symmetric four-qubit states $\psi_{S}^{(4)}$ which was presented by Bastin et al. [11]. Five socalled degeneracy configurations $\mathcal{D}_{\left\{n_{i}\right\}}$ define the five families of the SLOCC classification (see Table I).

The families $\mathcal{D}_{4}$ and $\mathcal{D}_{3,1}$ contain separable and $W$ states, respectively. All polynomial invariants vanish on those states, in analogy to the three-qubit case. For the representatives of the one-class families $\mathcal{D}_{2,2}$ and $\mathcal{D}_{2,1,1}$ all polynomials have identical values. That is, they cannot be distinguished by invariant polynomials alone although they are not SLOCC interconvertible. For the states in these families the invariant $\mathcal{C}$ depends on $\mathcal{A}$. According to Eq. (3) we can define a polynomial that vanishes for these families:

$$
\mathcal{D}=\mathcal{C}+\frac{5}{9} \mathcal{A}^{3}
$$

The family $\mathcal{D}_{1,1,1,1}$ contains a continuous parameter and thus infinitely many classes. It can be seen from Table I that 
TABLE I. Comparison of the polynomial characterization and the SLOCC classification of symmetric four-qubit states [11]. Note that for symmetric states the sum rule for the generators implies $\mathcal{B}^{j}=\mathcal{A}^{2}$. The representatives are given in the basis of the symmetric four-qubit Dicke states $D_{4}^{(k)}$ with $k|1\rangle$ components. For the continuous parameter in the $X$ family we have $\mu^{2} \neq 2 / 3$ and $a(\mu)=2+\mu^{2}, c(\mu)=-8+$ $4 \mu^{2}-\left(102 \mu^{4}+5 \mu^{6}\right) / 9$, and $d(\mu)=-8 / 9\left(2-3 \mu^{2}\right)^{2}$.

\begin{tabular}{lccccc}
\hline \hline $\mathcal{D}_{\left\{n_{i}\right\}}$ & Representative & $\mathcal{A}$ & $\mathcal{C}$ & $\mathcal{D}$ & Type \\
\hline $\mathcal{D}_{4}$ & $D_{4}^{(0)}$ & 0 & 0 & 0 & separable \\
$\mathcal{D}_{3,1}$ & $D_{4}^{(1)}$ & 0 & 0 & 0 & $W$ \\
$\mathcal{D}_{2,2}$ & $D_{4}^{(2)}$ & 1 & $-5 / 9$ & 0 & $D_{4}^{(2)}$ \\
$\mathcal{D}_{2,1,1}$ & $D_{4}^{(0)}+D_{4}^{(2)}$ & 1 & $-5 / 9$ & 0 & $D_{4}^{(2)}$ \\
$\mathcal{D}_{1,1,1,1}$ & $|0000\rangle+|1111\rangle+\mu D_{4}^{(2)}$ & $a(\mu)$ & $c(\mu)$ & $d(\mu)$ & $X$ \\
\hline \hline
\end{tabular}

$\mathcal{D}\left(\psi_{S}^{(4)}\right) \neq 0$ if and only if $\psi_{S}^{(4)} \in \mathcal{D}_{1,1,1,1}$. We term this $X$ type of entanglement after the $X$ state $[5,25]$

$$
\left|X_{4}\right\rangle=|0001\rangle+|0010\rangle+|0100\rangle+|1000\rangle+\sqrt{2}|1111\rangle .
$$

Consequently, for symmetric four-qubit states there is a hierarchy of SLOCC families which can be labeled by a "pattern" $\left[\mathcal{A}\left(\psi_{S}^{(4)}\right), \mathcal{D}\left(\psi_{S}^{(4)}\right)\right]$ that is obtained from two polynomial invariants. It is tempting to call these invariants "tangles." There are three levels in the hierarchy: $[\mathcal{A}=0, \mathcal{D}=0],[\mathcal{A} \neq$ $0, \mathcal{D}=0]$, and $[\mathcal{A}, \mathcal{D} \neq 0]$. We conclude the discussion of four-qubit symmetric states by noting that a hierarchy as in Table I is essential also for a classification of the corresponding mixed states. An example for symmetric $N$-qubit states (based on a different criterion) was given recently in Ref. [26].

\section{POLYNOMIALS AND GENERAL FOUR-QUBIT STATES}

These results raise the question whether the polynomial classification scheme can be extended beyond symmetric
TABLE III. The four-qubit SLOCC families defined via the tangle patterns of the invariants in Eq. (11). The invariant of highest nonvanishing degree determines the family to which a state belongs. We have named its entanglement type after a well-known state in the family.

\begin{tabular}{lccccc}
\hline \hline Type & $\mathcal{A}$ & $\mathcal{L}$ & $\mathcal{M}$ & $\mathcal{N}$ & $\mathcal{X}$ \\
\hline$W$ & 0 & 0 & 0 & 0 & 0 \\
GHZ & $A \neq 0$ & 0 & 0 & 0 & 0 \\
cluster & $A$ & $L \neq 0$ or & $M \neq 0$ & $-L-M$ & 0 \\
$X$ & $A$ & $L$ & $M$ & $-L-M$ & $X \neq 0$ \\
\hline \hline
\end{tabular}

states. Therefore, we inspect the SLOCC families in the classification due to Lamata et al. [8] (LLSS). In Table II we have listed all eleven representatives and corresponding tangle patterns for the eight LLSS families. Intriguingly, just as in the symmetric case, states from the same LLSS family show the same functional dependence between the polynomials, even for families containing infinitely many SLOCC classes. Those functional dependencies suggest a new grouping of the states according to the tangle pattern using the invariants [15]

$$
\begin{gathered}
\mathcal{L}=\frac{1}{48}\left(\mathcal{B}^{I I}-\mathcal{B}^{I I I}\right), \quad \mathcal{M}=\frac{1}{48}\left(\mathcal{B}^{I I I}-\mathcal{B}^{I}\right), \\
\mathcal{N}=\frac{1}{48}\left(\mathcal{B}^{I}-\mathcal{B}^{I I}\right), \\
\mathcal{X}=\left(\mathcal{C}+\mathcal{A}^{3}\right)^{2}-128 \mathcal{A}^{2}\left(\mathcal{L}^{2}+\mathcal{M}^{2}+\mathcal{N}^{2}\right),
\end{gathered}
$$

which remove the redundant functional dependencies. With these invariants we define a hierarchical ordering into four families according to the tangle pattern displayed in Table III. The choice of polymials (here $\mathcal{A}, \mathcal{L}, \mathcal{M}, \mathcal{N}, \mathcal{X}$ ) determines the classification one obtains. Each state $\psi^{(4)}$ is characterized by the numbers $\left[\mathcal{A}\left(\psi^{(4)}\right), \mathcal{L}\left(\psi^{(4)}\right), \mathcal{M}\left(\psi^{(4)}\right), \mathcal{X}\left(\psi^{(4)}\right)\right]$ where the nonvanishing polynomial of highest degree yields the family of the state. This is our central result.

TABLE II. Tangle patterns for the representatives of the SLOCC classification of Lamata et al. (cf. Table I in Ref. [8]). Here, $|\varphi\rangle, \ldots$, are single-qubit vectors with components $\left(\varphi_{0}, \varphi_{1}\right), \ldots$ The vectors $|\varphi\rangle$ and $|\bar{\varphi}\rangle, \ldots$, are linearly independent. Note that the representative in line 8

\begin{tabular}{|c|c|c|c|c|c|c|}
\hline LLSS family & Representative & $\mathcal{A}$ & $\mathcal{B}^{I}$ & $\mathcal{B}^{I I}$ & $\mathcal{B}^{I I I}$ & $\mathcal{C}$ \\
\hline $\mathcal{W}_{000,0_{k} \Psi}(\mathrm{b})$ & $|0000\rangle+|1101\rangle+|1110\rangle$ & 0 & 0 & 0 & 0 & 0 \\
\hline $\mathcal{W}_{000, W}$ & $|0001\rangle+|0010\rangle+|0100\rangle+|1000\rangle$ & 0 & 0 & 0 & 0 & 0 \\
\hline $\mathcal{W}_{000,000}$ & $|0000\rangle+|1111\rangle$ & 2 & 4 & 4 & 4 & -8 \\
\hline $\mathcal{W}_{000,0_{k} \Psi}(\mathrm{a})$ & $|0000\rangle+|1100\rangle+|1111\rangle$ & 2 & 4 & 4 & 4 & -8 \\
\hline $\mathcal{W}_{000, \mathrm{GHZ}}$ & $|0 \varphi \phi \psi\rangle+|1000\rangle+|1111\rangle$ & $2\left(\varphi_{0} \phi_{0} \psi_{0}-\varphi_{1} \phi_{1} \psi_{1}\right) \equiv A_{1}$ & $A_{1}^{2}$ & $A_{1}^{2}$ & $A_{1}^{2}$ & $-A_{1}^{3}$ \\
\hline $\mathcal{W}_{0_{k} \Psi, 0_{j} \Psi}(a)$ & $|0 \phi 00\rangle+|0 \phi 1 \psi\rangle+|1000\rangle+|1101\rangle$ & $-2\left(\phi_{0} \psi_{0}+\phi_{1} \psi_{1}\right) \equiv A_{2}$ & $A_{2}^{2}$ & $A_{2}^{2}$ & $A_{2}^{2}$ & $-A_{2}^{3}$ \\
\hline $\mathcal{W}_{0_{k} \Psi, 0_{j} \Psi}(b)$ & $|0 \phi 0 \psi\rangle+|0 \phi 10\rangle+|1000\rangle+|1101\rangle$ & $-2 \phi_{0} \equiv A_{3}$ & $A_{3}^{2}$ & $A_{3}^{2}$ & $A_{3}^{2}$ & $-A_{3}^{\overline{3}}$ \\
\hline \multirow{3}{*}{$\begin{array}{l}\mathcal{W}_{0_{k} \Psi, 0_{k} \Psi}(a) \\
\mathcal{W}_{0_{k} \Psi, 0_{k} \Psi}(b)\end{array}$} & $|0000\rangle+|1100\rangle+\lambda_{1}|0011\rangle+\lambda_{2}|1111\rangle$ & \multirow[t]{2}{*}{$2\left(\lambda_{1}+\lambda_{2}\right) \equiv A_{4}$} & \multirow[t]{2}{*}{$B_{4}^{I}$} & \multirow{2}{*}{$B_{4}^{I I} \neq B_{4}^{I}$} & \multirow[t]{2}{*}{$B_{4}^{I I}$} & \multirow[t]{2}{*}{$-A_{4} B_{4}^{I I}$} \\
\hline & $|0000\rangle+|1100\rangle+\lambda_{1}(|0001\rangle+|0010\rangle)$ & & & & & \\
\hline & $+\lambda_{2}(|1101\rangle+|1110\rangle)$ & $-4 \lambda_{1} \lambda_{2} \equiv A_{5}$ & $3 A_{5}^{2}$ & 0 & 0 & 0 \\
\hline $\mathcal{W}_{0_{k} \Psi, \mathrm{GHZ}}$ & $|0 \varphi\rangle \otimes(|\phi \psi\rangle+|\bar{\phi} \bar{\psi}\rangle)+|1000\rangle+|1111\rangle$ & $A_{6}$ & $B_{6}^{I}$ & $B_{6}^{I I} \neq B_{6}^{I}$ & $B_{6}^{I I}$ & $-A_{6} B_{6}^{I I}$ \\
\hline $\mathcal{W}_{\mathrm{GHZ}, W}$ & $|0001\rangle+|0010\rangle+|0100\rangle+|1 \varphi \phi \psi\rangle+|1 \bar{\varphi} \bar{\phi} \bar{\psi}\rangle$ & $A_{7}$ & $B_{7}^{I}$ & $B_{7}^{I I}$ & $B_{7}^{I I I}$ & $C_{7}$ \\
\hline
\end{tabular}
coincides with a cluster state for $\lambda_{1}=1, \lambda_{2}=-1$. The $X$ state is an element of the family in the last line. For brevity, the explicit expressions for $A_{6}, A_{7}, B_{4}^{I}, B_{4}^{I I}, \ldots, B_{7}^{I I}, C_{7}$ are omitted. Remarkably, we obtain precise functional dependencies between the polynomials for many of the LLSS families. 
Notice the apparent analogy between this hierarchy and the one for the symmetric states using the invariants $\mathcal{A}$ and $\mathcal{D}$, although the corresponding families certainly differ. This illustrates how the choice of the polynomials affects the grouping into families.

\section{DISCUSSION}

The analysis of the tangle patterns for two different SLOCC classifications has led us to a new SLOCC classification of four-qubit states based on polynomial invariants. It represents an independent classification method in its own right with several evident advantages.

(i) In contrast to all known SLOCC classifications it is straightforward to decide to which family a given arbitrary state belongs.

(ii) The tangle patterns characterize types of entanglement. Most strikingly, they provide not just a qualitative but even a quantitative description. According to Ref. [16], the modulus of any degree-2 invariant is an entanglement monotone [27,28]. That is, by choosing the absolute value of the appropriate power for each polynomial, the tangles of the pattern characterize quantitatively the types of multipartite entanglement contained in a given state.

(iii) Note that any (even incomplete) set of polynomials $[5,24]$ generates a corresponding classification. Our scheme displays a flexibility toward distinguishing certain desired properties: By comparing the classifications considered above one sees that an appropriate choice of polynomials can emphasize certain properties of the states in the families. It is particularly interesting that the polynomials of lowest degrees 2 and 4 separate peculiar states like GHZ and cluster states.

(iv) All these considerations can be extended to arbitrary multipartite systems with finite local dimensions.

\section{ACKNOWLEDGMENTS}

This work was supported by the German Research Foundation within SFB 631 (CE and OV), the German Academic Exchange Service (OV), the Nanosytems Initiative Munich (OV), and Basque Government Grant No. IT-472. The authors thank A. Osterloh for many stimulating discussions, L. Lamata for helpful comments, and K. Richter and J. Fabian for their support of this research. OV thanks the QUINST group in Bilbao for their hospitality.
[1] W. Dür, G. Vidal, and J. I. Cirac, Phys. Rev. A 62, 062314 (2000).

[2] J. Schlienz and G. Mahler, Phys. Rev. A 52, 4396 (1995); M. Grassl, M. Rötteler, and T. Beth, ibid. 58, 1833 (1998); H. A. Carteret et al., Found. Phys. 29, 527 (1999).

[3] F. Verstraete, J. Dehaene, B. DeMoor, and H. Verschelde, Phys. Rev. A 65, 052112 (2002).

[4] A. Miyake and F. Verstraete, Phys. Rev. A 69, 012101 (2004).

[5] A. Osterloh and J. Siewert, Phys. Rev. A 72, 012337 (2005).

[6] L. Lamata and J. Leon, Phys. Rev. A 73, 052322 (2006).

[7] A. Mandilara, V. M. Akulin, A. V. Smilga, and L. Viola, Phys. Rev. A 74, 022331 (2006).

[8] L. Lamata, J. Leon, D. Salgado, and E. Solano, Phys. Rev. A 75, 022318 (2007).

[9] Y. Cao and A. M. Wang, Eur. Phys. J. D 44, 159 (2007).

[10] O. Chterental and D. Ž. Đoković, in Linear Algebra Research Advances, edited by G. D. Ling (Nova Science, Hauppauge, NY, 2007), Chap. 4, pp. 133-167.

[11] T. Bastin, S. Krins, P. Mathonet, M. Godefroid, L. Lamata, and E. Solano, Phys. Rev. Lett. 103, 070503 (2009).

[12] L. Borsten, D. Dahanayake, M. J. Duff, A. Marrani, and W. Rubens, Phys. Rev. Lett. 105, 100507 (2010).

[13] D. F. Li, X. R. Li, H. T. Huang et al., Quant. Inf. Comput. 9, 778 (2009).
[14] B. Kraus, Phys. Rev. Lett. 104, 020504 (2010).

[15] J.-G. Luque and J.-Y. Thibon, Phys. Rev. A 67, 042303 (2003).

[16] F. Verstraete, J. Dehaene, and B. De Moor, Phys. Rev. A 68, 012103 (2003).

[17] S. Hill and W. K. Wootters, Phys. Rev. Lett. 78, 5022 (1997).

[18] V. Coffman, J. Kundu, and W. K. Wootters, Phys. Rev. A 61, 052306 (2000).

[19] A. Wong and N. Christensen, Phys. Rev. A 63, 044301 (2001).

[20] M. S. Leifer, N. Linden, and A. Winter, Phys. Rev. A 69, 052304 (2004).

[21] G. Gour, Phys. Rev. Lett. 105, 190504 (2010).

[22] J.-L. Brylinski and R. Brylinski, in Mathematics of Quantum Computation, edited by G. Chen and R.-K. Brylinski (Chapman and Hall/CRC Press, London, 2002), Chap. 11.

[23] J. Eisert and H. J. Briegel, Phys. Rev. A 64, 022306 (2001).

[24] D. Ž. Đoković and A. Osterloh, J. Math. Phys. 50, 033509 (2009).

[25] A. Osterloh and J. Siewert, New J. Phys. 12, 075025 (2010).

[26] T. Bastin, P. Mathonet, and E. Solano, e-print arXiv:1011.1243.

[27] V. Vedral, M. B. Plenio, M. A. Rippin, and P. L. Knight, Phys. Rev. Lett. 78, 2275 (1997).

[28] G. Vidal, J. Mod. Opt. 47, 355 (2000). 